\title{
SModelS extension with the CMS supersymmetry search results from Run 2
}

\author{
Juhi Dutta, ${ }^{1}$, Sabine Kraml, ${ }^{2}$, Andre Lessa, ${ }^{3}$ and Wolfgang Waltenberger ${ }^{4}$ \\ ${ }^{1}$ Regional Centre for Accelerator-based Particle Physics, Harish-Chandra Research Institute, HBNI, Chhatnag Road, Jhusi, \\ Allahabad-211019, India \\ ${ }^{2}$ Laboratoire de Physique Subatomique et de Cosmologie, Université Grenoble-Alpes, CNRS/IN2P3, 53 Avenue des Martyrs, F-38026 \\ Grenoble, France \\ ${ }^{3}$ Centro de Ciências Naturais e Humanas, Universidade Federal do ABC, Santo André, 09210-580 SP, Brazil \\ ${ }^{4}$ Institut für Hochenergiephysik, Österreichische Akademie der Wissenschaften, Nikolsdorfer Gasse 18, 1050 Wien, Austria \\ Received: 10 March 2018, Accepted: 6 April 2018, Published: 12 May 2018
}

\begin{abstract}
We present the update of the SModelS database with the simplified model results from CMS searches for supersymmetry at Run 2 with $36 \mathrm{fb}^{-1}$ of data. The constraining power of these new results is compared to that of the $8 \mathrm{TeV}$ results within the context of a full model, the pMSSM. The new database, v1.1.2, is publicly available and can readily be employed for physics studies with SModelS.
\end{abstract}

DOI: 10.31526/LHEP.1.2018.02

Simplified models [1, 2, 3, 4, 5] have become one of the standard methods for ATLAS and CMS to optimise analyses for specific signatures, compare the reach, and communicate the results of their searches for new particles. When simplified model results are provided in terms of cross section upper limits or efficiency maps, they can readily be re-used to constrain arbitrary beyond-the-standard-model (BSM) theories in which the same final state occurs, as long as differences in the event kinematics (e.g., from different production mechanisms or from the spin of the BSM particle) do not significantly affect the signal acceptance of the experimental analysis. This is precisely the idea behind SModelS [6, 7].

SModelS is a public tool which allows to exploit the plethora of constraints on simplified model spectra (SMS) from ATLAS and CMS searches for supersymmetry (SUSY) in an automatised way. The principle of SModelS, in the current version 1.1 , is to decompose BSM collider signatures featuring a $\mathbb{Z}_{2}$ symmetry into simplified model topologies, using a generic procedure where each SMS is defined by the vertex structure and the SM final state particles; BSM particles are described only by their masses, production cross sections and branching ratios ${ }^{2}$ The working principle is illustrated in Fig. 11 The SModelS code and database are publicly available on GitHub at https://github.com/SModelS/ or on the SModelS wiki page, http://smodels.hephy.at/.

The previous database version [8] (v1.1.1) was comprised of 186 results (125 upper limits and 61 efficiency maps) from 21 ATLAS and 23 CMS SUSY searches, covering a total of 37 simplified models. From these 44 searches, the vast majority were based on Run 1 data. Only 11 (4 from ATLAS and 7 from CMS) were based on early $13 \mathrm{TeV}$ Run 2 data with $2-13 \mathrm{fb}^{-1}$ of integrated luminosity; most of these were preliminary results from ATLAS conference notes or CMS public analysis summaries.

\footnotetext{
Email address: sabine.kraml@lpsc.in2p3.fr (Sabine Kraml, ${ }^{2}$ )

${ }^{1}$ C) 2018 The Author(s). Published by LHEP. This is an open access article under the CC BY licensehttp://creativecommons .org/licenses/by/4.0/

${ }^{2}$ The $\mathbb{Z}_{2}$ symmetry is important because it determines the structure of the signal topologies: $\mathbb{Z}_{2}$-odd particles are produced in pairs and cascade-decay to the lightest one, which is stable. See $6[$ for details.
}

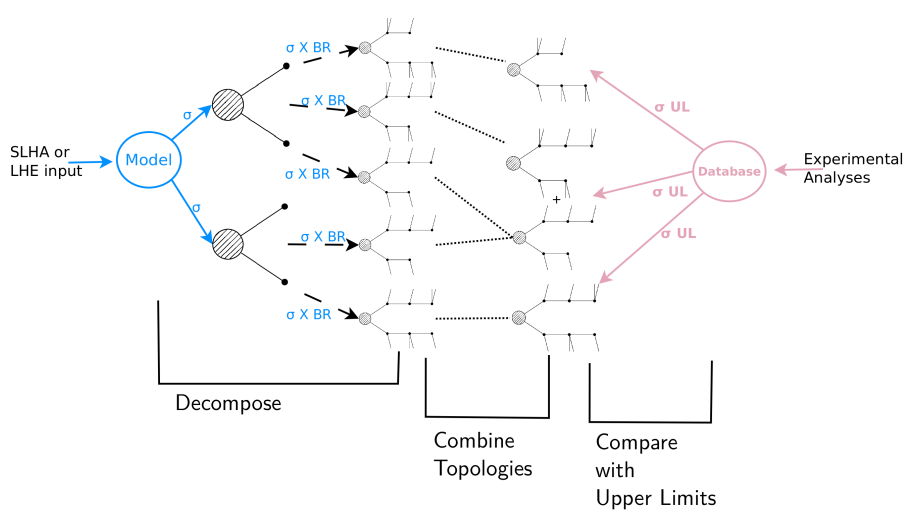

FIGURE 1: Schematic view of the working principle of SModelS.

In this note we now present the implementation of the Run 2 SUSY search results from CMS with $36 \mathrm{fb}^{-1}$, presented at the Moriond and the summer (LHCP and EPS) conferences of 2017. This extends the SModelS database by 84 new cross section upper limit (UL) maps from 19 different analyses. We give an overview which results have been included, show their validation in SModelS, and demonstrate their constraining power for the phenomenological Minimal Supersymmetric Standard Model (pMSSM) as compared to the $8 \mathrm{TeV}$ data.

The v1.1.2 database presented here includes results from 19 CMS SUSY analyses from Run 2 with $36 \mathrm{fb}^{-1}$ of data, comprising in total 84 new SMS results for the full 2016 dataset ${ }^{3}$ A detailed list is given in Table 1

All these new CMS results are upper limit maps: they give the $95 \%$ confidence level (CL) upper limit values on $\sigma \times \mathrm{BR}$ for a particular SMS as a function of the relevant parameters, usually the SUSY particle masses or slices over mass planes. They are derived from the colour maps in the simplified model limit plots of the experimental papers, which CMS systematically provides in numerical form, typically as

${ }^{3}$ Analogous results from ATLAS have become available on HEPData and will be added as soon as possible. 


\begin{tabular}{|c|c|c|c|c|}
\hline & Analysis & Ref. & ID & SMS results (txnames) \\
\hline \multirow{13}{*}{ 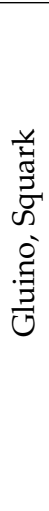 } & jet multiplicity $+H_{T}^{\text {miss }}$ & 9 & SUS-16-033 & T1, T1bbbb, T1tttt, \\
\hline & & & & $\mathrm{T} 2, \mathrm{~T} 2 \mathrm{bb}, \mathrm{T} 2 \mathrm{tt}$ \\
\hline & jets $+E_{T}^{\text {miss }}, M_{T 2}$ & [10] & SUS-16-036 & $\mathrm{T} 1, \mathrm{~T} 1 \mathrm{bbbb}, \mathrm{T} 1 \mathrm{tttt}$ \\
\hline & & & & $\mathrm{T} 2, \mathrm{~T} 2 \mathrm{bb}, \mathrm{T} 2 \mathrm{tt}, \mathrm{T} 2 \mathrm{cc}$ \\
\hline & & & & $\mathrm{T}^{6} \mathrm{bbWW} \mathrm{W}^{\dagger}$ \\
\hline & 1 lept. + jets $+E_{T}^{\text {miss }}, M_{J}$ & [11 & SUS-16-037 & $\mathrm{T} 1 \mathrm{tttt}, \mathrm{T} 5 \mathrm{tttt}^{\dagger}$ \\
\hline & 1 lept. + jets $+E_{T}^{\text {miss }}, \Delta \Phi$ & [12] & SUS-16-042 & T1tttt, T5WW ${ }^{\dagger}$ \\
\hline & 2 OS lept. + jets $+E_{T}^{\text {miss }}$ & [13] & SUS-16-034 & $\mathrm{T} \mathrm{ZZ} \mathrm{Z}^{+}, \mathrm{TChiWZ}$ \\
\hline & 2 SS lept. + jets $+E_{T}^{\text {miss }}$ & [14] & SUS-16-035 & T1tttt, T5WW ${ }^{\dagger}$, T5ttbbWW ${ }^{\dagger}$ \\
\hline & & & & $\mathrm{T} \mathrm{tttt}^{\dagger}, \mathrm{T} 5 \mathrm{tctc}{ }^{\dagger}, \mathrm{T} 6 \mathrm{ttWW}{ }^{\dagger}$ \\
\hline & multi-lept. + jets $+E_{T}^{\text {miss }}$ & [15] & SUS-16-041 & $\mathrm{T} 1 \mathrm{tttt}, \mathrm{T} 6 \mathrm{HH} \mathrm{tt}^{\dagger}, \mathrm{T} 6 \mathrm{ZZtt}{ }^{\dagger}$ \\
\hline & & & & $\mathrm{T} 6 \mathrm{tt} W \mathrm{~W}^{\dagger}$ \\
\hline & 0 lept. + top tag & [16 & SUS-16-050 & $\mathrm{T} 1 \mathrm{tttt}, \mathrm{T} 2 \mathrm{tt}, \mathrm{T} 5 \mathrm{tttt}^{\dagger}, \mathrm{T} 5 \mathrm{tctc}{ }^{\dagger}$ \\
\hline \multirow{5}{*}{ 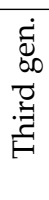 } & 0 lepton stop & [17] & SUS-16-049 & T2tt, T2ttC, T2cc, T6bbWW ${ }^{\dagger}$ \\
\hline & 1 lepton stop & [18] & SUS-16-051 & $\mathrm{T} 2 \mathrm{tt}, \mathrm{T} 6 \mathrm{bbWW} \mathrm{W}^{\dagger}$ \\
\hline & 2 lepton stop & $\overrightarrow{19}$ & SUS-17-001 & $\mathrm{T} 2 \mathrm{tt}, \mathrm{T} 6 \mathrm{bbWW}{ }^{+}$ \\
\hline & $b$ or $c$-jets $+E_{T}^{\text {miss }}$ & [20] & SUS-16-032 & $\mathrm{T} 2 \mathrm{bb}, \mathrm{T} 2 \mathrm{cc}$ \\
\hline & soft lepton, compressed stop & [21] & PAS-SUS-16-052 & T2bbWWoff, T6bbWWoff ${ }^{+}$ \\
\hline \multirow{6}{*}{ 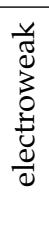 } & $W H(H \rightarrow b \bar{b})+E_{T}^{\text {miss }}$ & 22 & SUS-16-043 & TChiWH \\
\hline & multi-leptons $+E_{T}^{\text {miss }}$ & 23 & SUS-16-039 & TChiWH, TChiWZ, \\
\hline & & & & TChiChipmSlepL, \\
\hline & & & & TChiChipmSlepStau, \\
\hline & & & & TChiChipmStauStau \\
\hline & EWK combination & [24] & PAS-SUS-17-004 & TChiWH, TChiWZ \\
\hline \multirow{3}{*}{ 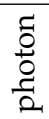 } & Razor $+H \rightarrow \gamma \gamma$ & [25] & SUS-16-045 & TChiWH, T6bbHH $^{\dagger}$ \\
\hline & photon $+E_{T}^{\text {miss }}$ & [26] & SUS-16-046 & T5gg, T6gg \\
\hline & photon $+H_{T}$ & 27] & SUS-16-047 & T5gg, T6gg \\
\hline
\end{tabular}

TABLE 1: CMS $13 \mathrm{TeV}$ results for $36 \mathrm{fb}^{-1}$ included in this SModelS database update. The last column lists the specific SMS results included, using the shorthand "txname" notation (see text for details). For brevity, only the on-shell results are listed, although the off-shell ones are always also included (e.g., T1tttt in the table effectively means T1tttt and T1ttttoff). The superscript † denotes SMS with three mass parameters, for which only one mass plane is available; we included them for completeness but note that they apply to the given $2 \mathrm{D}$ slice of parameter space only, not to general mass patterns.

ROOT files on the analyses' wiki pages $4^{4}$ Each included map is thoroughly validated to make sure it reproduces the limits reported in the experimental publication. Figure 2 shows some examples of validation plots; the full set is available online at http://smodels.hephy.at/wiki/Validationv112.

We note that a few results in Table 1 are from CMS PASes, i.e. they are preliminary results; these will be updated to the final published results in a future release.

Inside SModelS, individual SMS results are identified by the analysis ID and the txname (see right-most column in Table 1 , which describes in a shorthand notation the hypothesised SUSY process used to derive the UL map. The txnames largely follow the notation introduced in [5]. For instance, 'T1' topologies stand for gluino-pair production followed by 3body gluino decay into the lightest SUSY particle (LSP), usually the $\tilde{\chi}_{1}^{0}$, hence:

$$
\begin{aligned}
& \text { T1: } p p \rightarrow \tilde{g} \tilde{g}, \tilde{g} \rightarrow q \bar{q} \tilde{\chi}_{1}^{0}, \\
& \text { T1bbbb: } p p \rightarrow \tilde{g} \tilde{g}, \tilde{g} \rightarrow b \bar{b} \tilde{\chi}_{1}^{0}, \\
& \text { T1tttt: } p p \rightarrow \tilde{g} \tilde{g}, \tilde{g} \rightarrow t \bar{t} \tilde{\chi}_{1}^{0}, \ldots
\end{aligned}
$$

\footnotetext{
${ }^{4}$ Alternatively, SModelS v.1.1 can also use efficiency maps 8. Efficiency maps (EMs) have the advantage that contributions from different topologies to the same signal region can be combined.
}

T1bbtt would mean one gluino decays into $b \bar{b} \tilde{\chi}_{1}^{0}$ and the other one into $t \bar{t} \tilde{\chi}_{1}^{0}$, but results for such asymmetric topologies are currently not available. 'T5' also stands for gluino-pair production but with the decay proceeding via an intermediate onshell SUSY particle (for example T5tttt: $p p \rightarrow \tilde{g} \tilde{g}, \tilde{g} \rightarrow t \tilde{t}_{1}$, $\left.\tilde{t}_{1} \rightarrow t \tilde{\chi}_{1}^{0}\right)$. Along the same lines, 'T2' and 'T6' denote squark $(\tilde{q}, \tilde{b}, \tilde{t})$ pair production followed by, respectively, direct or cascade decay into the LSP (e.g., T2tt: $p p \rightarrow \tilde{t} \tilde{t}, \tilde{t} \rightarrow t \tilde{\chi}_{1}^{0}$; T6bbWW: $\left.p p \rightarrow \tilde{t} \tilde{t}, \tilde{t} \rightarrow b \tilde{\chi}_{1}^{+}, \tilde{\chi}_{1}^{+} \rightarrow W \tilde{\chi}_{1}^{0}\right)$. A complete list of $t x-$ names and the corresponding diagrams can be consulted at http://smodels.hephy.at/wiki/SmsDictionary

We note also that, whenever relevant, the experimental results for topologies with top quarks and/or massive gauge bosons are split into several UL maps according to different kinematic regions where the tops, Ws or Zs are on-shell or off-shell. For example, an experimental result for $p p \rightarrow \tilde{t} \tilde{t}$, $\tilde{t} \rightarrow t \tilde{\chi}_{1}^{0}$ will have two UL maps in SMODELS, one called T2tt covering the region where the $\Delta m=m_{\tilde{t}}-m_{\tilde{\chi}_{1}^{0}} \geq m_{t}-2 \Gamma_{t}$, $\Gamma_{t}$ being the top total width, and one called T2ttoff covering $m_{W}<\Delta m<m_{t}-2 \Gamma_{t}$. The reason is that for T2tt the final state to be constrained is $2 t+E_{T}^{\text {miss }}$ while for T2ttoff it is $2 b 2 W+E_{T}^{\text {miss }}$. (Below $\Delta m=m_{W}$, one enters a different regime of stop 4-body or loop decays.) The $2 b 2 W+E_{T}^{\text {miss }}$ final state also 

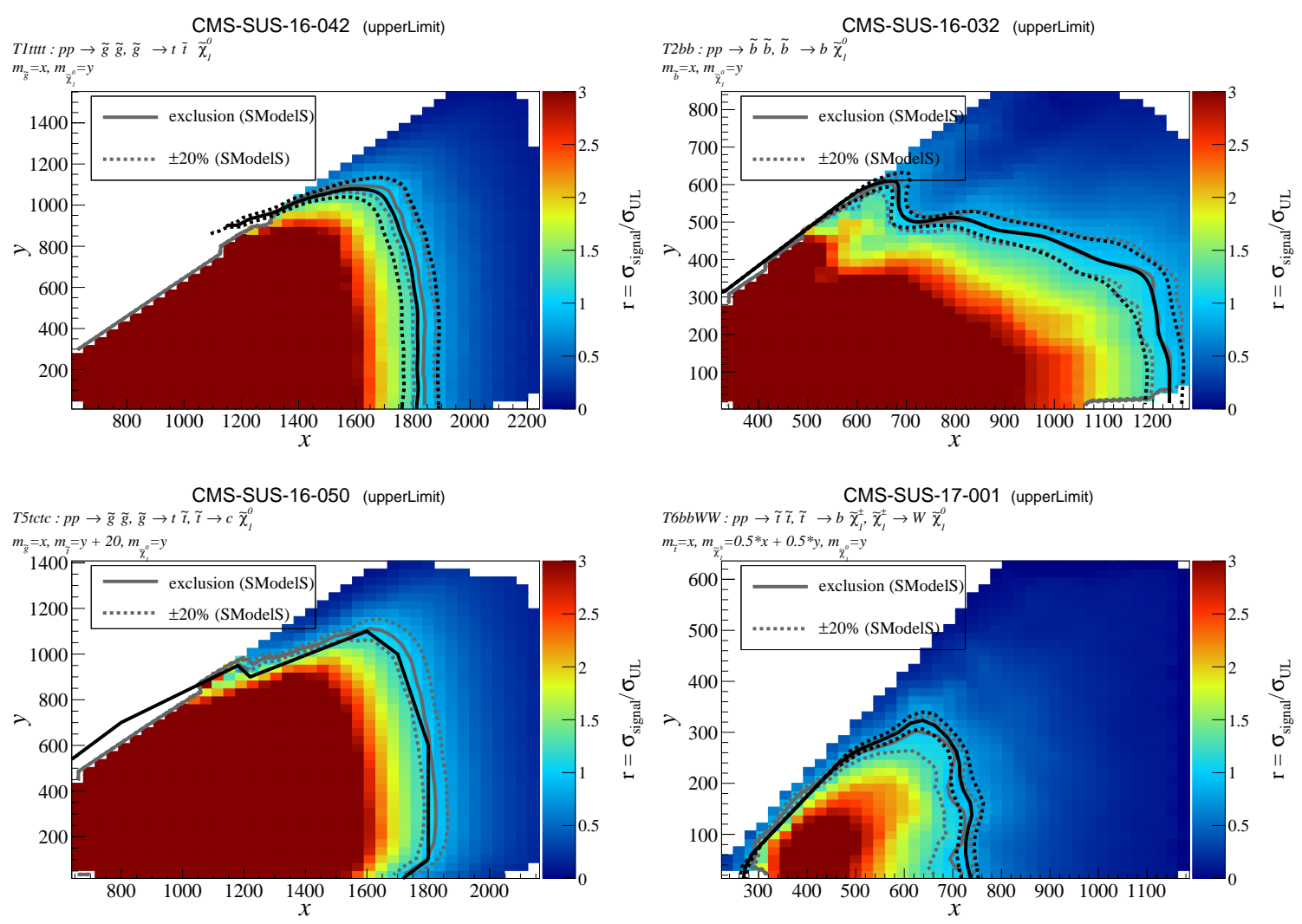

FIGURE 2: Examples of validation plots. The coloured histograms show the $r$-values, defined as the ratio of the theory prediction over the observed upper limit. The full gray lines are the SModelS exclusion contours, $r=1$, to be compared to the official CMS exclusion lines in black ( $\pm 1 \sigma$ as dashed black lines). The effect of a $20 \%$ variation, $r=1 \pm 0.2$, is indicated by the dashed grey lines.

arises from stop decays via a chargino, $\tilde{t} \rightarrow b \tilde{\chi}_{1}^{+} \rightarrow b W^{+} \tilde{\chi}_{1}^{0}$, but this has a different topology (vertex structure) and corresponds to a distinct simplified model (T6bbWW). For conciseness, the "off" maps are not listed in Table 1, with the exception of PASSUS-16-052, which has only UL maps for compressed spectra where Ws are always off-shell.

In total, the 84 new results in the v1.1.2 database cover 25 distinct topologies (35 when counting on- and off-shell versions separately). As can also be seen in Table 1. several analyses have SMS interpretations for the same topologies (txnames). For instance, upper limits for $p p \rightarrow \tilde{g} \tilde{g}, \tilde{g} \rightarrow t \bar{t} \tilde{\chi}_{1}^{0}$ (T1tttt) are provided in seven of the eight searches for gluinos. Likewise, the different stop searches in the 0,1 , and 2 leptons channels all give upper limits for $p p \rightarrow \tilde{t}_{1} \tilde{t}_{1}, \tilde{t}_{1} \rightarrow t \tilde{\chi}_{1}^{0}$ (T2tt) and $p p \rightarrow \tilde{t}_{1} \tilde{t}_{1}$, $\tilde{t}_{1} \rightarrow b \tilde{\chi}_{1}^{+} \rightarrow b W \tilde{\chi}_{1}^{0}$ (T6bbWW). In principle it would be possible to compile, for each topology, the limits from different analyses into one single map, using only the strongest constraint in each mass bin. Instead, we have chosen to include all the individual results which are provided by the experimental collaboration. This makes the database larger and the evaluation slightly slower, but has the advantage of more flexibility. For instance it allows to compare the constraining power of different analyses for the same signal. When speed is a limiting factor, knowledgeable users can build a slimmed-down pickle file, applying only the subset of analyses which give the strongest constraints; see the SModelS v1.1 manual [8] for more details.

There is a further reason for including all individual SMS results: when using SModelS to constrain non-SUSY scenarios, it is possible that, depending on the selection cuts in the analyses, some SMS results do not apply. Such results should then be disregarded. Generally, the validity of the SMS assumptions depends on the concrete model under consideration, as well as details of the experimental search. It is the responsibility of the user to verify this case by case when testing new theories. In practice this means verifying that the signal acceptance as function of the BSM masses is approximately the same for the new model as for the model assumed in the experimental paper. This can be done by explicitly recasting the experimental analysis for a few benchmark points and/or by comparing the relevant kinematic distributions in the new theory and the model which underlies the SMS result.

To assess the impact of these new $13 \mathrm{TeV}$ results in a general manner, we make use of the extensive scan of the pMSSM [28] with 19 free parameters from the ATLAS pMSSM study [29] (see also [30, 31, 32, 33]).

The ATLAS collaboration made the whole scan, in total more than $310 \mathrm{k}$ parameter points with SUSY masses up to $4 \mathrm{TeV}$, publicly available on HepData [34]. These points were classified into three sets according to the nature of the LSP: bino-like $(103,410$ points), wino-like $(80,233$ points $)$ and higgsino-like $\left(126,684\right.$ points). They all have $m_{h}=[124,128] \mathrm{GeV}$ and satisfy constraints from SUSY searches at LEP and the Tevatron, flavor and electroweak precision measurements, cold dark matter relic density and direct dark matter searches. We remove from this dataset the points which contain long lived charged sparticles $(c \tau>1 \mathrm{~mm})$, which cannot be treated in the official 


\begin{tabular}{l|c|c|c} 
& Bino-like LSP & Higgsino-like LSP & Wino-like LSP \\
\hline Total number of points & 99,492 & 123,498 & 8,772 \\
\hline \# points excluded - 8 TeV results only & 23,253 & 32,219 & 1,389 \\
\hline \# points excuded - full database & 62,159 & 65,768 & 3,212
\end{tabular}

TABLE 2: Summary of results, listing the total number of points tested from the ATLAS pMSSM scan (without long-lived charged particles), the number of points excluded by SModelS using only the $8 \mathrm{TeV}$ database and the number of points excluded when using the full database with 8 $\mathrm{TeV}$ and $13 \mathrm{TeV}$ results.
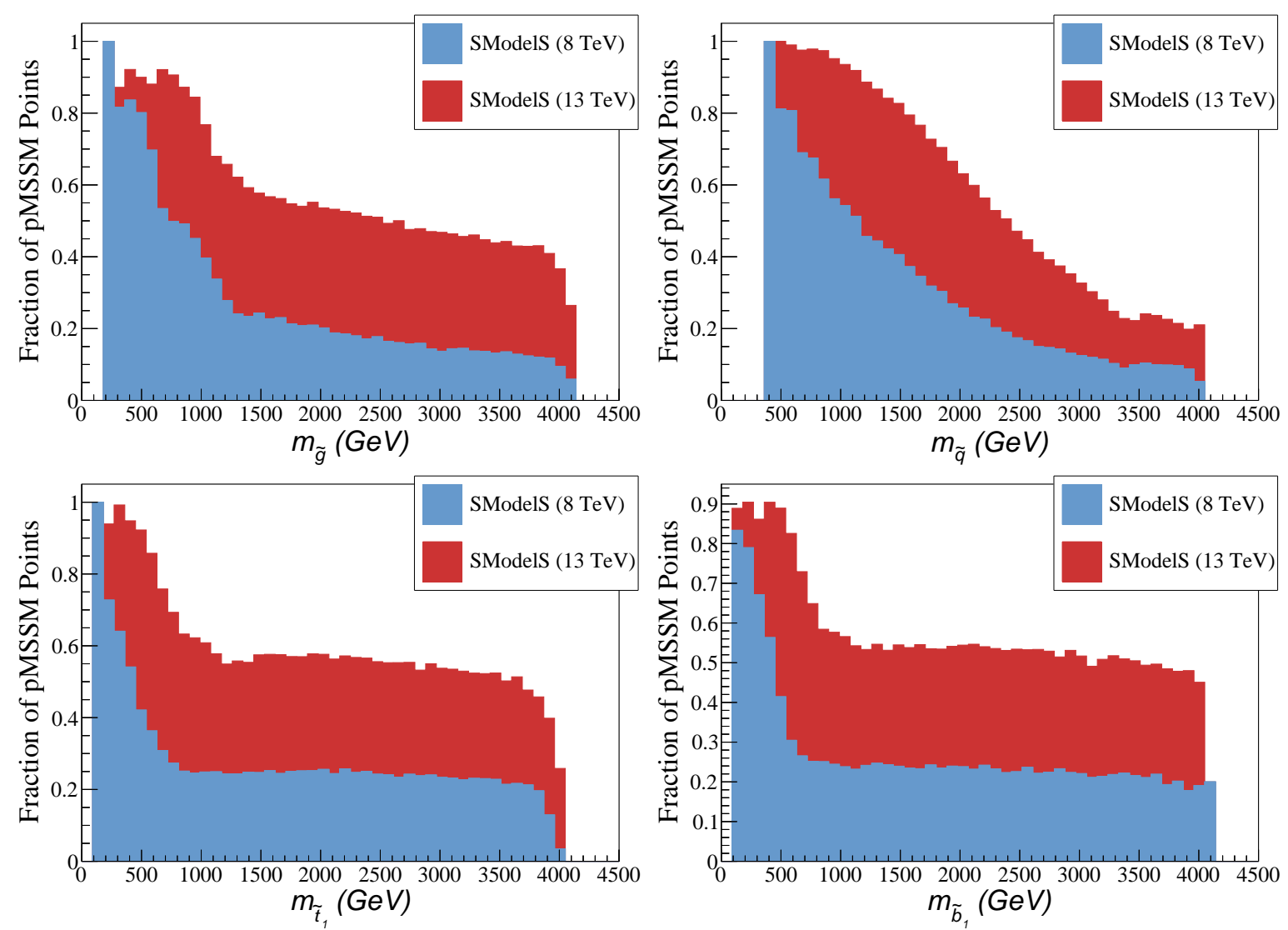

FIGURE 3: Fraction of points excluded by SModelS for the ATLAS pMSSM scan as a function of gluino, average squark, stop and sbottom mass. Only the points without long-lived charged particles were considered. The blue histogram shows the fraction of excluded points using only the $8 \mathrm{TeV}$ database, while the red histogram shows the increase of excluded points once the $13 \mathrm{TeV}$ database is included.

SModelS version. This has only a small effect on the bino-like and higgsino-like LSP sets $(99,492$ and 123,498 points remaining, respectively) but removes most of the wino-like LSP points (only 8,772 points remaining).

For this dataset, we analyse how the SModelS exclusion improves with the new $13 \mathrm{TeV}$ results as compared to the $8 \mathrm{TeV}$ results. As a first overview, we list in Table 2 the total number of points studied, the number of points that can be excluded by SModelS when using only the $8 \mathrm{TeV}$ results in the database, and the number of points that can be excluded when using the full $8 \mathrm{TeV}+13 \mathrm{TeV}$ database. As one can see, the gain is quite substantial, between a factor of 2 for the higgsino-like LSP dataset and a factor of 2.7 for the bino-like dataset.

The impact on the gluino, average squark, stop and sbottom masses is illustrated in Fig. 3. We see that gluinos with masses below 1 (1.5) TeV are now much better constrained, with only about $11 \%(22 \%)$ of points escaping exclusion by sim- plified model results in this mass range. Likewise, the SMS constraints are severely closing in on stops, sbottoms and lightflavor squarks, with around $70 \%$ of points with at least one squark below $1 \mathrm{TeV}$ being excluded. Also interesting is the impact on the LSP mass, shown in Fig. 4 The $8 \mathrm{TeV}$ results eliminate about $54 \%$ of the pMSSM points with LSP masses below about $100 \mathrm{GeV}$, but show a steep drop in constraining power for heavier $\tilde{\chi}_{1}^{0}$. The new $13 \mathrm{TeV}$ results, on the other hand, provide strong constraints for $\tilde{\chi}_{1}^{0}$ masses up to about $600 \mathrm{GeV}$, excluding $64 \%$ of the pMSSM points in this range and more than $75 \%$ of the points with $m_{\tilde{\chi}_{1}^{0}} \lesssim 100 \mathrm{GeV}$.

To address the question which signal topologies are most relevant for the improved constraints, Fig. 5 provides a breakdown by txnames as a function of the gluino mass. For each point excluded at $13 \mathrm{TeV}$, but not at $8 \mathrm{TeV}$, we take the txname with the highest $r$-value $\left(r=\sigma_{\mathrm{SMS}} / \sigma_{\mathrm{UL}}\right)$ and then show the 


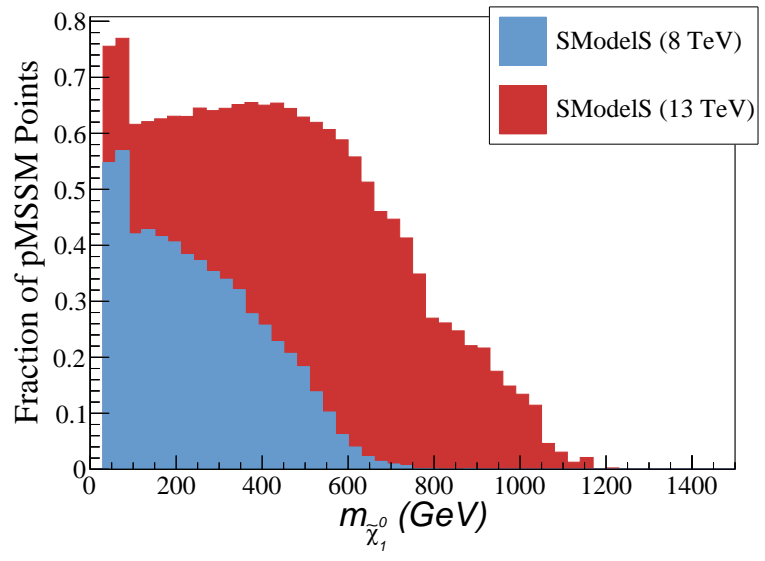

FIGURE 4: As Fig. 3 but for the neutralino LSP mass.

(stacked) histograms for each txname normalized by the total number of points in each bin.

As we can see, when gluinos are within reach (i.e., for $m_{\tilde{g}} \lesssim 1.5 \mathrm{TeV}$ ), T1 and T1bbbb are among the most constraining topologies for the bino-like and wino-like datasets; for higgsino-like LSP dataset gluino decays via the 3rd generation are more important and thus T1bbbb and T1ttt are among the most constraining topologies. Overall, however, and increasingly so at high gluino masses, the strongest exclusion comes from squark topologies. Indeed, T2 (2 jets $\left.+E_{T}^{\text {miss }}\right)$ is clearly the leading topology with some contribution also from T2bb (2bjets $+E_{T}^{\text {miss }}$ ).

A comment is in order regarding the prominence of the T2cc topology in the bino-like LSP dataset. In principle, T2cc describes stop-pair production followed by stop decays into $c+\tilde{\chi}_{1}^{0}$. However, from the three analyses [10], 17, 20] which provide constraints for this case, only [20] includes charm tagging. The other two [10, 17] actually constrain 2 jets $+E_{T}^{\text {miss }}$, not $2 c$-jets $+E_{T}^{\text {miss }}$, so they also apply to what is normally a T2 topology, i.e. $p p \rightarrow \tilde{q} \tilde{q}, \tilde{q} \rightarrow q \tilde{\chi}_{1}^{0}$ or $p p \rightarrow \tilde{g} \tilde{g}, \tilde{g} \rightarrow g \tilde{\chi}_{1}^{0}$. We note here that the conventional T2 UL maps cover squarkLSP mass differences down to $25 \mathrm{GeV}$ only. The UL maps for T2cc from [10, 17], on the other hand, are designed to cover the compressed region and go down to mass differences of 11-12 GeV. Furthermore, for mass differences $\lesssim 80 \mathrm{GeV}$, the $\mathrm{T} 2 \mathrm{cc}$ results are more constraining than the T2 results. They can therefore considerably extend the exclusion of points with one light squark or gluino close in mass to the LSP.

\section{DOWNLOAD AND INSTALLATION}

The full v.1.1.2 database is now included in the SModelS v1.1 release available on GitHub at https://github.com/SModelS/ or on the SModelS homepage, http://smodels . hephy . at/ Installation instructions are given in the manual, available as paper $[8]$ and online, and the INSTALLATION.rst file in the distribution.

For people who have already installed SModelS, smodels-database-v1.1.2.tar.gz is also available separately from

http://smodels.hephy.at/wiki/CodeReleases.
For the standard installation, it suffices to put this tarball into the main smodels folder and explode it there. That is, the following steps need to be performed

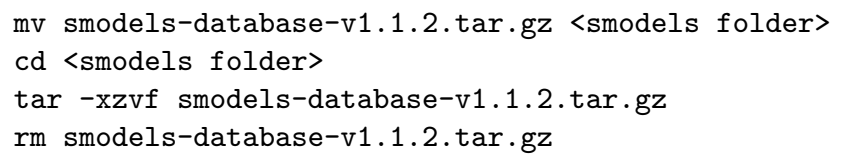

The v1.1.2 database will be unpacked into the smodels-database directory, replacing the previous version and the pickle file will then be automatically rebuilt on the next run of SModelS. For a clean installation, it is recommended to first remove the previous database version. If the tarball is unpacked to another location, one has to correctly set the SModelS database path when running SModelS. If using runSModels.py, this is done in the parameters.ini file.

Alternatively, the database can also be obtained from the https://github.com/SModelS/smodels-database-release repository.

We presented the update of the SModelS database with the simplified model cross section upper limits from 19 CMS SUSY analyses from Run 2 with $36 \mathrm{fb}^{-1}$ of data. These results significantly improve previously available constraints. Using the pMSSM as a showcase for a realistic model, we demonstrated how the limits on various SUSY masses are pushed to higher values by the $13 \mathrm{TeV}$ results as compared to $8 \mathrm{TeV}$ results. The improved constraints affect not only the masses of colored sparticles-particularly noticeable are the much stronger constraints on LSP masses up to about $600 \mathrm{GeV}$. All in all, the number of points from the ATLAS pMSSM scan [29] which can be excluded by SModelS increases by a factor 2.3 as compared to the $8 \mathrm{TeV}$ results.

The v1.1.2 database is publicly available and can readily be used in SModelS to constrain arbitrary BSM models which have a $\mathbb{Z}_{2}$ symmetry, provided the SMS assumptions [6, 8] apply. The simplified model results from ATLAS searches for $36 \mathrm{fb}^{-1}$ at $13 \mathrm{TeV}$ available on HEPData will be included as soon as possible.

\section{ACKNOWLEDGEMENTS}

We thank the CMS SUSY group for providing a vast amount of SMS cross section upper limits in digital format. Moreover, we owe special thanks to Federico Ambrogi for his contribution in the early stage of this work.

J.D. is partially supported by funding available from the Department of Atomic Energy, Government of India, for the Regional Centre for Accelerator-based Particle Physics (RECAPP), Harish-Chandra Research Institute. She thanks moreover the LPSC Grenoble for hospitality, and the INFREHEPNET (IndoFrench Network on High Energy Physics) of CEFIPRA/IFCPAR (Indo-French Centre for the Promotion of Advanced Research), as well as the "Investissements d'avenir, Labex ENIGMASS" for financial support for a research visit in May 2017, during which this work was started.

S.K. acknowledges support from the IN2P3 project "Théorie - LHCiTools" and the CNRS-FAPESP collaboration PRC275431. A.L. acknowledges support by the São Paulo Research Foundation (FAPESP), projects 2015/20570-1 and 2016/50338-6.

A couple of results from the CMS publications listed in Table 1 have not been implemented in the v1.1.2 database, because they cannot be re-used well in SModelS. This is notably 

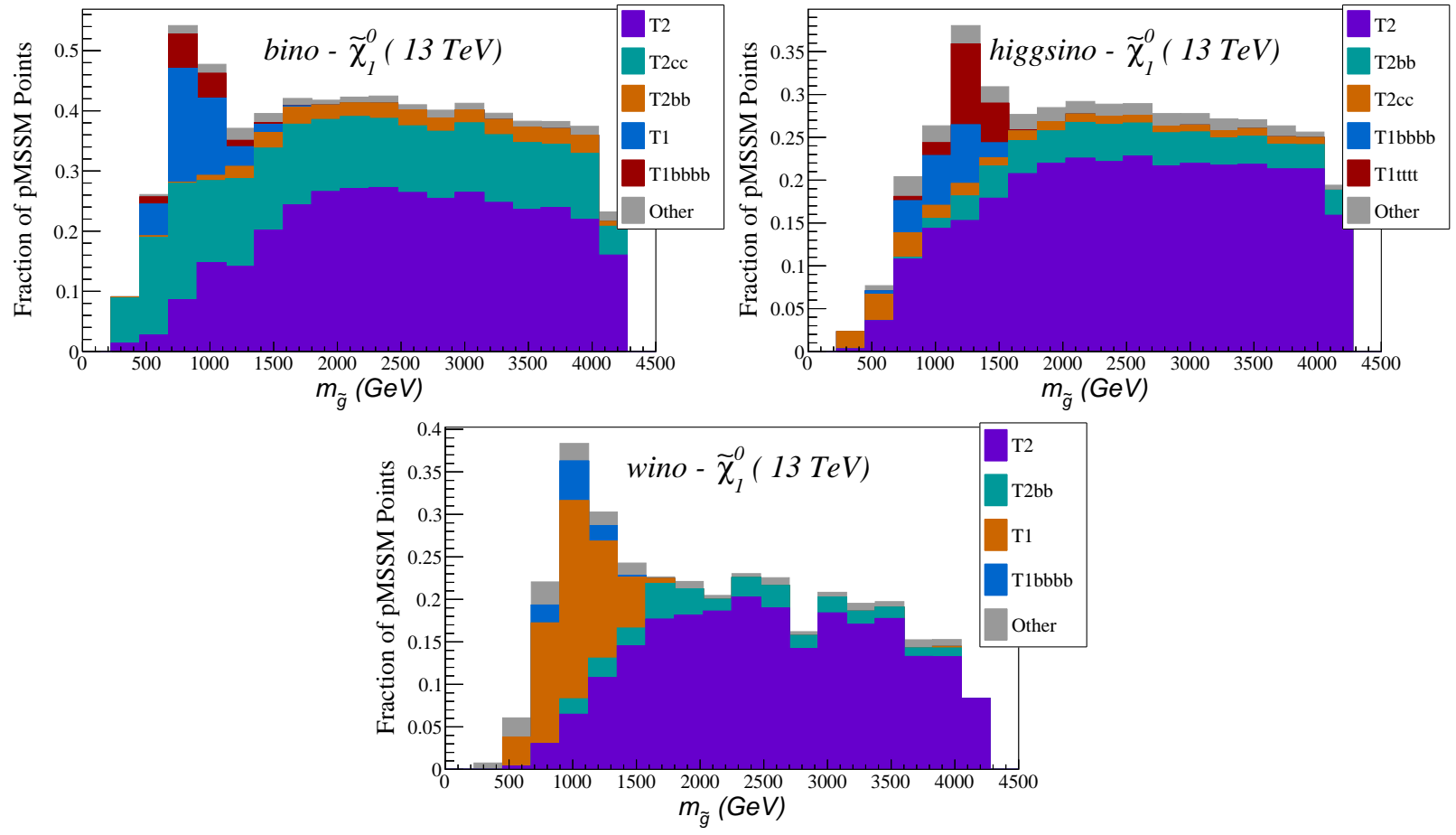

FIGURE 5: Fraction of points excluded by SModelS for the ATLAS pMSSM scan as a function of the gluino mass for the binoLSP, higgsino-LSP and wino-LSP scenarios. Only the points without long-lived charged particles were considered. The colored histograms show the topologies which give the highest exclusion.

the case for SMS results with mixed decay modes, where different intermediate $\mathbb{Z}_{2}$-odd particles and/or different final states are summed over. They pose constraints on a very specific sum of topologies, which is not applicable to the general case. Examples are:

- Fig. 12d in SUS-16-033 and Fig. 5b in SUS-16-041: these are constraints on gluino-pair production followed by $\tilde{g} \rightarrow q \bar{q} \tilde{\chi}_{1}^{ \pm} \rightarrow q \bar{q} W \tilde{\chi}_{1}^{0}$ and $\tilde{g} \rightarrow q \bar{q} \tilde{\chi}_{2}^{0} \rightarrow q \bar{q} Z \tilde{\chi}_{1}^{0}$ decays with $50 \%$ branching ratio each. CMS treats this as a T5VV $(\mathrm{V}=W, \mathrm{Z})$ topology. For SModelS, however, this results represents an UL map for the weighted sum of three topologies, $25 \% \mathrm{~T} 5 \mathrm{WW}+25 \% \mathrm{~T} 5 \mathrm{ZZ}+50 \% \mathrm{~T} 5 \mathrm{WZ}$.

- Fig. 8b in SUS-16-036, Fig. 9 in SUS-16-049 and Fig. 7 in SUS-16-051: these are limits on stop-pair production for $\mathrm{BR}\left(\tilde{t} \rightarrow b \tilde{\chi}_{1}^{+}\right)=\operatorname{BR}\left(\tilde{t} \rightarrow t \tilde{\chi}_{1}^{0}\right)=0.5$. As in the bullet item above, the UL applies to the weighted sum of three topologies, 25\% T2tt $+25 \% \mathrm{~T} 2 \mathrm{bb}+50 \% \mathrm{~T} 2 \mathrm{tb}$;

- The T5Wg results in SUS-16-046 and SUS-16-047: these are actually a sum over T5WW, T5gg and T5Wg for a given branching ratio;

As discussed in [35], results for asymmetric topologies, arising from two different decays happening on the two branches of the topology diagram, would be very useful to improve the constraining power of SMS results. In principle one could try to interpolate between the UL maps for the symmetric topologies with $100 \%$ BR and the ones for $50 \%$ BRs including the mixed topologies. This would add a level of complication in the matching with the decomposition procedure, which is the most time-consuming part of the calculation. Moreover, the validation of such a procedure would require full recasting, as there are no official results for intermediate BRs to compare to. Much better and simpler would be if efficiency maps for the individual symmetric and asymmetric topologies were available. This would allow to work out the limits for arbitrary branching ratios in a fast, reliable and robust way.

Another class of results which are not included are long cascade decays (with more than one intermediate particle) where intermediate masses are fixed and/or branching ratios summed over. An example is Fig. 10 of SUS-16-034. Here, pairproduced sbottoms decay via $\tilde{b}_{1} \rightarrow b \tilde{\chi}_{2}^{0}$ followed by $\tilde{\chi}_{2}^{0} \rightarrow$ $l^{ \pm} \tilde{l}^{\mp} \rightarrow l^{+} l^{-} \tilde{\chi}_{1}^{0}$ or $\tilde{\chi}_{2}^{0} \rightarrow Z^{(*)} \tilde{\chi}_{1}^{0}$. From the SModelS point of view this is not a simplified model topology.

Finally, the results of a number of newer CMS publications or public analysis summaries are not included, because the ROOT files for the SMS limits are not yet available. This concerns the analyses SUS-16-048 [36] (2 soft leptons), SUS-17-003 [37 (hadronic staus), and the searches in leptonic final states presented at the SUSY 2017 conference SUS-17-002 [38] and SUS-17-009 [39]. They will be added later when the relevant ROOT files are available.

\section{References}

[1] N. Arkani-Hamed, P. Schuster, N. Toro, J. Thaler, L.-T. Wang, B. Knuteson, and S. Mrenna, "MARMOSET: The Path from LHC Data to the New Standard Model via On-Shell Effective Theories," hep-ph/0703088.

[2] J. Alwall, P. Schuster, and N. Toro, "Simplified Models for a First Characterization of New Physics at the LHC," Phys. Rev. D79 (2009) 075020, 0810.3921

[3] LHC New Physics Working Group Collaboration, D. Alves, "Simplified Models for LHC New Physics 
Searches," J. Phys. G39 (2012) 105005, 1105.2838

[4] ATLAS Collaboration, H. Okawa, "Interpretations of SUSY Searches in ATLAS with Simplified Models," in Particles and fields. Proceedings, Meeting of the Division of the American Physical Society, DPF 2011, Providence, USA, August 9-13, 2011. 2011. 1110.0282

[5] CMS Collaboration, S. Chatrchyan et al., "Interpretation of Searches for Supersymmetry with simplified Models," Phys. Rev. D88 (2013), no. 5, 052017, 1301.2175

[6] S. Kraml, S. Kulkarni, U. Laa, A. Lessa, W. Magerl, D. Proschofsky-Spindler, and W. Waltenberger, "SModelS: a tool for interpreting simplified-model results from the LHC and its application to supersymmetry," Eur. Phys. J. C74 (2014) 2868, 1312.4175

[7] S. Kraml, S. Kulkarni, U. Laa, A. Lessa, V. Magerl, W. Magerl, D. Proschofsky-Spindler, M. Traub, and W. Waltenberger, "SModelS v1.0: a short user guide," 1412.1745

[8] F. Ambrogi, S. Kraml, S. Kulkarni, U. Laa, A. Lessa, V. Magerl, J. Sonneveld, M. Traub, and W. Waltenberger, "SModelS v1.1 user manual: Improving simplified model constraints with efficiency maps," Comput. Phys. Comm. (in press) 1701.06586

[9] CMS Collaboration, A. M. Sirunyan et al., "Search for supersymmetry in multijet events with missing transverse momentum in proton-proton collisions at 13 TeV," Phys. Rev. D96 (2017), no. 3, 032003, 1704.07781

[10] CMS Collaboration, A. M. Sirunyan et al., "Search for new phenomena with the $M_{\mathrm{T} 2}$ variable in the all-hadronic final state produced in proton?proton collisions at $\sqrt{s}=13 \mathrm{TeV}$," Eur. Phys. J. C77 (2017), no. 10, $710,1705.04650$

[11] CMS Collaboration, A. M. Sirunyan et al., "Search for Supersymmetry in $p p$ Collisions at $\sqrt{s}=13 \mathrm{TeV}$ in the Single-Lepton Final State Using the Sum of Masses of Large-Radius Jets," Phys. Rev. Lett. 119 (2017), no. 15, 151802, 1705.04673

[12] CMS Collaboration, A. M. Sirunyan et al., "Search for supersymmetry in events with one lepton and multiple jets exploiting the angular correlation between the lepton and the missing transverse momentum in proton-proton collisions at $\sqrt{s}=13 \mathrm{TeV}, " 1709.09814$

[13] CMS Collaboration, A. M. Sirunyan et al., "Search for new phenomena in final states with two opposite-charge, same-flavor leptons, jets, and missing transverse momentum in pp collisions at $\sqrt{s}=13 \mathrm{TeV}$," 1709.08908

[14] CMS Collaboration, A. M. Sirunyan et al., "Search for physics beyond the standard model in events with two leptons of same sign, missing transverse momentum, and jets in proton?proton collisions at $\sqrt{s}=13 \mathrm{TeV}$," Eur. Phys. J. C77 (2017), no. 9, 578, 1704.07323

[15] CMS Collaboration, A. M. Sirunyan et al., "Search for supersymmetry in events with at least three electrons or muons, jets, and missing transverse momentum in proton-proton collisions at $\sqrt{s}=13 \mathrm{TeV}, " 1710.09154$

[16] CMS Collaboration, A. M. Sirunyan et al., "Search for supersymmetry in proton-proton collisions at $13 \mathrm{TeV}$ using identified top quarks," Phys. Rev. D97 (2018), no. 1, 012007, 1710.11188

[17] CMS Collaboration, A. M. Sirunyan et al., "Search for direct production of supersymmetric partners of the top quark in the all-jets final state in proton-proton collisions at $\sqrt{s}=13 \mathrm{TeV}$," JHEP 10 (2017) 005, 1707.03316

[18] CMS Collaboration, A. M. Sirunyan et al., "Search for top squark pair production in pp collisions at $\sqrt{s}=13 \mathrm{TeV}$ using single lepton events," JHEP 10 (2017) 019, 1706.04402 .

[19] CMS Collaboration, A. M. Sirunyan et al., "Search for top squarks and dark matter particles in opposite-charge dilepton final states at $\sqrt{s}=13 \mathrm{TeV}, " 1711.00752$

[20] CMS Collaboration, A. M. Sirunyan et al., "Search for the pair production of third-generation squarks with two-body decays to a bottom or charm quark and a neutralino in proton-proton collisions at $\sqrt{(s)}=13 \mathrm{TeV}$," 1707.07274

[21] CMS Collaboration, "Search for supersymmetry in events with at least one soft lepton, low jet multiplicity, and missing transverse momentum in proton-proton collisions at $\sqrt{s}=13 \mathrm{TeV}$," Tech. Rep.

CMS-PAS-SUS-16-052, CERN, Geneva, 2017.

[22] CMS Collaboration, A. M. Sirunyan et al., "Search for electroweak production of charginos and neutralinos in WH events in proton-proton collisions at $\sqrt{s}=13 \mathrm{TeV}$," JHEP 11 (2017) 029, 1706.09933

[23] CMS Collaboration, A. M. Sirunyan et al., "Search for electroweak production of charginos and neutralinos in multilepton final states in proton-proton collisions at $\sqrt{s}=13 \mathrm{TeV}$, , 1709.05406

[24] CMS Collaboration, "Combined search for electroweak production of charginos and neutralinos in pp collisions at $\sqrt{s}=13 \mathrm{TeV}, "$ Tech. Rep. CMS-PAS-SUS-17-004, CERN, Geneva, 2017.

[25] CMS Collaboration, A. M. Sirunyan et al., "Search for supersymmetry with Higgs boson to diphoton decays using the razor variables at $\sqrt{s}=13 \mathrm{TeV}, " 1709.00384$

[26] CMS Collaboration, A. M. Sirunyan et al., "Search for gauge-mediated supersymmetry in events with at least one photon and missing transverse momentum in pp collisions at $\sqrt{s}=13 \mathrm{TeV}, " 1711.08008$

[27] CMS Collaboration, A. M. Sirunyan et al., "Search for supersymmetry in events with at least one photon, missing transverse momentum, and large transverse event activity in proton-proton collisions at $\sqrt{s}=13$ TeV," JHEP 12 (2017) 142, 1707.06193

[28] MSSM Working Group Collaboration, A. Djouadi et al., "The Minimal supersymmetric standard model: Group summary report," in GDR (Groupement De Recherche) Supersymetrie, Montpellier, France, April 15-17, 1998. hep-ph/9901246

[29] ATLAS Collaboration, G. Aad et al., "Summary of the ATLAS experiment's sensitivity to supersymmetry after LHC Run 1 interpreted in the phenomenological MSSM," JHEP 10 (2015) 134, 1508.06608

[30] C. F. Berger, J. S. Gainer, J. L. Hewett, and T. G. Rizzo, "Supersymmetry Without Prejudice," JHEP 02 (2009) 023, 0812.0980

[31] M. W. Cahill-Rowley, J. L. Hewett, S. Hoeche, A. Ismail, and T. G. Rizzo, "The New Look pMSSM with Neutralino and Gravitino LSPs," Eur. Phys. J. C72 (2012) 2156, 1206.4321

[32] M. W. Cahill-Rowley, J. L. Hewett, A. Ismail, and T. G. Rizzo, "More energy, more searches, but the phenomenological MSSM lives on," Phys. Rev. D88 
(2013), no. 3, 035002, 1211.1981

[33] M. Cahill-Rowley, J. L. Hewett, A. Ismail, and T. G. Rizzo, "Lessons and prospects from the pMSSM after LHC Run I," Phys. Rev. D91 (2015), no. 5, 055002, 1407.4130.

[34] http://hepdata.cedar.ac.uk/view/ins1389857

[35] F. Ambrogi, S. Kraml, S. Kulkarni, U. Laa, A. Lessa, and W. Waltenberger, "On the coverage of the pMSSM by simplified model results," 1707.09036 .

[36] CMS Collaboration, A. M. Sirunyan et al., "Search for new physics in events with two soft oppositely charged leptons and missing transverse momentum in proton-proton collisions at $\sqrt{s}=13 \mathrm{TeV}, " 1801.01846$

[37] CMS Collaboration Collaboration, "Search for pair production of tau sleptons in $\sqrt{s}=13 \mathrm{TeV}$ pp collisions in the all-hadronic final state," Tech. Rep.

CMS-PAS-SUS-17-003, CERN, Geneva, 2017.

[38] CMS Collaboration Collaboration, "Search for supersymmetry in events with tau leptons and missing transverse momentum in proton-proton collisions at sqrt(s)=13 TeV," Tech. Rep. CMS-PAS-SUS-17-002, CERN, Geneva, 2017.

[39] CMS Collaboration Collaboration, "Search for selectrons and smuons at $\sqrt{s}=13 \mathrm{TeV}$," Tech. Rep. CMS-PAS-SUS-17-009, CERN, Geneva, 2017. 